\title{
Los entes de razón en Suárez. Una concepción barroca de la realidad
}

\author{
The beings of reason in Suarez. \\ A baroque conception of the reality
}

\author{
Óscar BARRoso FernÁNDEZ \\ Universidad de Granada \\ obarroso@ugr.es
}

Recibido: 10/12/2010

Aceptado: 15/01/2011

\section{Resumen}

En el presente artículo se pretende mostrar la importante función que Suárez tiene reservada a los entes de razón: el aseguramiento de la validez científica. Al respecto resulta fundamental caer en la cuenta de la diferencia entre la quimera y el resto de los entes de razón: negación, relación de razón y privación. Con ello Suárez ha asentado las bases de la manera propiamente barroca de entender el mundo: una realidad extramental para cuyo conocimiento científico requerimos de la introducción del artificio, del ente de razón; aunque Suárez tiene aún en mente el modelo de ciencia aristotélica, basado en la relación de universalidad, lo que le hace despreciar los entes de razón matemáticos. En todo caso, creemos que los entes de razón ayudan a comprender la singularidad de la metafísica barroca respecto al ontologismo posterior. Nos situamos así, críticamente, frente a las interpretaciones marcadamente ontologistas de la metafísica de Suárez.

Palabras clave: Francisco Suárez, metafísica, ontología, ente de razón, Barroco.

\section{Abstract}

In this paper I shall show the relevant role which the beings of reason play in Suarez's philosophy, namely, the role of being the guarantee of the scientific valid- 
ity. For this thesis it is basic to discover the difference between the chimera and other beings of reason: the negation, the relation of reason and the privation. In this way, Suarez sets the basis of the properly baroque way of understanding the world as an extra-mental reality which is only cognoscible through the artful device of the being of reason. Suarez conceives the science from the Aristotelean perspective, that is why he does not appreciate the mathematical beings of reason. Nevertheless, we do believe that the notion of being of reason is helpful in the comprehension of the singularity of the baroque metaphysics in comparison to the posterior ontologism. We enter, therefore, in discussion with the marked tendency to interpret the metaphysics of Suarez in an ontological way.

Key words: Francisco Suarez, metaphysics, ontology, being of reason, Baroque

\section{Introducción}

En el siglo XX se han propuesto diversas lecturas de la obra de Suárez coincidentes en hacer hincapié en la importancia que su metafísica tuvo para la constitución de la ontología moderna ${ }^{1}$. Sin duda destacan al respecto la interpretación esencialista de Gilson ${ }^{2}$ y la ontológica de Heidegger ${ }^{3}$. Esta última ha alcanzado su máximo desarrollo en las investigaciones sobre la metafísica de Suárez llevadas a cabo por Courtine 4 .

\footnotetext{
${ }^{1} \mathrm{He}$ analizado las distintas interpretaciones que se han ofrecido en el siglo XX de la ontología de Suárez en "Suárez, filósofo de encrucijada o del nacimiento de la ontología", Pensamiento, 232 (2006), pp. 121-138. También puede verse mi trabajo sobre la interpretación heideggeriana de las Disputationes: "De la metafísica creacionista a la ontología objetivista. La interpretación heideggeriana de las Disputationes Metaphysicae de Francisco Suárez", en L. Saez, J. de la Higuera y J. Zúñiga (eds.), Pensar la nada. Ensayos sobre filosofia y nihilismo, Madrid, Biblioteca Nueva, 2007, pp. 6584. Para el estudio de las interpretaciones de la metafísica de Suárez es también muy recomendable el trabajo de Costantino Esposito: "Ritorno a Suárez. Le Disputationes Metaphysicae nella critica contemporánea”, en Ada Lamacchia (ed.), La filosofia nel siglo de oro. Studi sul tardo rinacimento spagnolo, Bari, Levante Editori, 1995, pp. 466-573. Respecto a la interpretación en concreto de Heidegger, pueden consultarse también los siguientes trabajos: C. G. Noreña, "Heidegger, on Suárez: the 1927 Marburg Lectures", International Philosophical Quarterly, 23 (1983) 407-442; C. Esposito, "Heidegger, Suárez e la storia dell'ontologia", Quaestio. Annuario di storia della metafisica, 1 (2001) 407-430.

2 E. Gilson, Index scolastico-cartésien, Alcan, Paris, 1913; L'être et l'essence, Paris, Vrin, 1981 (1ª ed. 1948); El ser y los filósofos, Pamplona, Eunsa, 1979 (1ª ed. orig. 1949).

3 M. Heidegger, Die Grundprobleme der Phänomenologie, Gesamtausgabe, vol. 24, Frankfurt, Klostermann, 1975, pp. 108-171 (trad. esp. J.J. García Norro, Los problemas fundamentales de la fenomenología, Madrid, Trotta, 2000, pp. 109-157); Die Grundbegriffe der Metaphysik. Welt, Endlichkeit, Einsamkeit, Gesamtausgabe, vols. 29/30, Frankfurt, Klostermann, 1983, § 13-14 (trad. esp. Alberto Ciria, Los conceptos fundamentales de la metafisica. Mundo, finitud, soledad, Madrid, Alianza, 2007, pp. 73-84).

4 J. F. Courtine, Suarez et le système de la métaphysique, Paris, PUF, 1990.
} 
En su edición original, las Disputationes Metaphysicae aparecían divididas en dos volúmenes. Por un lado, las disputaciones I-XXVII constituyen lo que podríamos denominar, en lenguaje wolffiano, una ontología general. En ella se define y delimita la noción de 'ente', objeto adecuado de la metafísica (disputaciones I y II); se analizan las propiedades trascendentales del ente y todos los problemas relacionados con este asunto (disputaciones III-XI), y se estudian los principios y causas del ente (disputaciones XII-XXVII). Por otro lado, hay una segunda parte que queda claramente al margen de esta ontología general y que constituye un conglomerado de elementos de lo que después se llamarán ontologías especiales: una teología natural -que incluye la necesaria distinción y comparación del ente finito y del infinito- (disputaciones XXVIII-XXX); y un estudio del ente finito que parte de la tradicional división del ente en sustancia y accidente, y que desemboca en el estudio de los predicamentos (disputaciones XXXI-LIII). La obra se completa con un estudio de los entes de razón, cuyo estudio queda justificado, en principio, como necesario para entender correctamente en qué consiste un ente real (disputación LIV), aunque nuestro objetivo aquí va a ser precisamente mostrar que tal justificación es a todas luces insuficiente.

Si nos fijamos en las interpretaciones ontológicas desarrolladas en el siglo XX, constatamos que han tenido como objeto fundamental de análisis el primer volumen de las Disputationes Metaphysicae, es decir, aquello que de alguna forma constituye en Suárez la propuesta de una metafísica general. Desde esta perspectiva, el tránsito de una metafísica del acto a una ontología objetiva parece más que justificado. Aunque esta interpretación no carece de problemas.

El ontologismo de Suárez tiene un freno realista inviolable inherente a la propia definición de la metafísica que nos dio en la primera de las disputaciones: es "la ciencia que considera al ente en cuanto tal, o en cuanto que prescinde de la materia según el ser" $(1,3,1)^{5}$. Mientras que la primera parte de la definición apunta al ámbito de lo ontológico, la segunda, seu in quantum a materia abstrahit secundum esse contemplatur, se refiere a lo óntico. De aquí se puede concluir que la metafísica depende de la existencia de sustancias inmateriales, en el sentido de que si estas no existieran, la metafísica se volvería superflua. A ello apunta directamente el siguiente pasaje:

Quitada esta sustancia, se arrebataría justamente el objeto adecuado y propio de la filosofía primera, porque no sólo desaparecería la sustancia inmaterial, sino también todas las razones de ente o de sustancia, comunes a las cosas materiales e inmateriales, y en

\footnotetext{
5 Las citas de las Disputationes metaphysicae se harán siguiendo la traducción de Sergio Rábade, Salvador Caballero y Antonio Puiggerver, Disputaciones metafisicas, Madrid, Gredos, 1960-66. Citaremos en el cuerpo del texto, utilizando, entre paréntesis, tres números, que indican, sucesivamente, la disputación, la sección y el parágrafo.
} 
esta hipótesis, al no haber ningunos entes inmateriales, tampoco habría ningunas razones de ente que prescindiesen de la materia según el ser y, por consiguiente, no habría necesidad de ninguna ciencia distinta $(1,1,16)$.

Sólo una vez que hemos afirmado el objeto real de la metafísica a través de la postulación de sustancias inmateriales, ésta puede hacerse cargo también de todas las razones de ente abstractas. Pero no podemos perder de vista que para Suárez no tendría sentido una ciencia cuyo objeto estuviera totalmente desvinculado de la realidad extramental. Suárez concluye al respecto afirmando que la metafísica

abstrae de la materia sensible e inteligible, no sólo según la razón, sino según el ser, porque las razones de ente que considera se hallan en la realidad sin materia y, por tanto, en su concepto propio y objetivo de por sí no incluye la materia $(1,2,13)$.

La abstracción secundum esse es lo que hace que la metafísica sea ciencia y no mera lógica. Si la metafísica es sabiduría, no puede serlo sólo en tanto que se hace cargo del ente en cuanto ente, porque la noción abstracta de ente tiene sólo una perfección mínima si la comparamos con la de cualquier ente determinado, por ínfimo que sea. Y para Suárez es claro que para la razón de sabiduría no basta la dignidad de objeto cognoscible si falta la de las cosas que se conocen $(1,5,15)$.

Por lo tanto, si la metafísica está por encima del resto de las ciencias, no es sólo porque se haga cargo de la noción más abstracta de ente, sino porque trata de los seres inmateriales y, entre ellos, del primero y principal: Dios.

A grandes rasgos, podemos decir que la metafísica suareciana se encuentra a medio camino entre dos formas de entender la perfección: la medieval, que considera esta perfección desde un punto de vista existencial, entendiendo que son más perfectos los seres que existen efectivamente; y la moderna, que encuentra la perfección en la certeza y la simplicidad.

La metafísica sigue así dependiente del ámbito trascendente, aún cuando este ámbito permanezca subordinado, en orden a la estructura interna de la filosofía primera, al ámbito trascendental. Si no hubiera realidades inmateriales, las razones más abstractas podrían ser estudiadas por la filosofía natural; pero al haberlas, esta filosofía no puede hacerse cargo de las razones más comunes, ya que son comunes al ser material y al inmaterial.

Por lo demás, aquí encontramos la razón por la que en Suárez la ontología regional no puede incluir una filosofía de la naturaleza y una antropología. En el primer caso, porque coincidiría con el orden material, y el en segundo porque el alma racional "dice esencial orden a la materia" $(1,2,19)$, y por ello "ha de ser relegada a la última y más perfecta parte de la filosofía natural" $(1,2,20)$.

En todo caso, no es mi objetivo en el presente trabajo enfrentar la interpretación ontológica de Suárez, pero era necesario este rodeo para alcanzar un planteamiento 
adecuado de los ens rationis. Creo que una cabal interpretación de la metafísica de Suárez debe intentar mantenerse en los marcos que establece al respecto la contrarreforma jesuita. Sólo desde ella son comprensibles los cambios que Suárez introduce y que permiten entender su metafísica como profundamente barroca y, por lo tanto, moderna ${ }^{6}$. Desde la perspectiva ontologista muchos de estos elementos son vistos sólo como impurezas teológicas 7 . Con ello se minusvalora la importancia que el orden existencial sigue teniendo en la propuesta metafísica de Suárez. Desde la interpretación ontologista ha sido habitual entender que Suárez ha dado lugar a una nueva noción de realidad entendida no desde el ámbito de la causación, sino desde el de la intención objetiva. En la perspectiva de Courtine, la clave de bóveda para pensar el giro efectuado por Suárez está en el carácter ontológicamente fundamental del extra nihil del aliquid respecto a su extra causas, es decir, respecto a su existir efectivo ${ }^{8}$. La oposición más radical será la que se da entre nihil y aliquid, con lo que el aliquid en el sentido de non-nihil se constituirá en el primer concepto positivo de la ontología; la entitas minima9. Con ello, nos dice Courtine, la ontología se ha constituido independientemente como ciencia primera, pasando la teología natural a constituir una ciencia esencialmente regional. Por ello, cuando Suárez pretende definir el objeto adecuado de la metafísica -y de acuerdo con la tradición escotista- se orienta hacia las communis ratio entis. Desde esta perspectiva, si Dios tiene un sitio en la metafísica, será solamente porque se deja englobar bajo esta noción

\footnotetext{
${ }^{6} \mathrm{He}$ analizado los caracteres generales del Barroco jesuita a partir de la metafísica de Suárez en "La metafísica de Suárez en la filosofía barroca", en Congreso Internacional Andalucía Barroca. IV. Ciencia, Filosofía y Religión, Junta de Andalucía, 2009, pp. 17-26. Otro trabajo interesante al respecto es: E. Rivera de Ventosa, "El barroco español dentro de la cultura europea", Cuadernos Salmantinos de Filosofia, 16 (1989), pp. 89-105; Por lo demás, el estudio del Barroco filosófico ha de resultar hoy fundamental habida cuenta de las diferentes e incluso contrapuestas lecturas que de él se han propuesto en las últimas décadas. Efectivamente, si por un lado tenemos lecturas positivas del barroco, que incluso entienden que su estudio ha de resultar fundamental para una adecuada comprensión de los problemas de nuestra época, como es el caso de la obra de Deleuze El pliegue. Leibniz y el Barroco (Barcelona, Paidós, 1989, $1^{\text {a }}$ ed. orig. 1988); por otro lado nos encontramos con lecturas negativas de esta etapa histórica, que de alguna forma entienden que la superación de las contradicciones de nuestro presente histórico pasan por la deconstrucción de los cambios que se produjeron entre el Renacimiento y el Barroco. Este es el caso de Foucault en Las palabras y las cosas (Madrid, Siglo XXI, 2005, $1^{\text {a }}$ ed. orig. 1968) y Hardt y Negri en Imperio (Barcelona, Paidós, 2002, $1^{\text {a }}$ ed. orig. 2000). 7 M. Forlivesi, “Ontologia impura. La natura della metafisica secondo Francisco Suárez", en VV.AA., Francisco Suárez: 'Der ist der Mann'. Homenaje al Prof. Salvador Castellote, Valencia, Facultad de Teología San Vicente Ferrer, 2004, pp. 161-207.

8 Suarez et le système de la métaphysique, pp. 246-292. También J. F. Courtine, "Le proyet suarézien de la métaphysique, pour une étude de la thèse suarézienne du néant", Archives de Philosophie, 42 (1979), pp. 235-274.

9 De esta forma, la ambigüedad del ser extra causas y el ser non-nihil llegará, al menos, hasta Baumgarten para quien el aliquid, en cuanto non-nihil, constituye con propiedad, en su determinación, al ente, aunque en otro sentido, en cuanto nihil privativum, es un mero posible. Baumgarten, Metaphysica, Halle/Salle, 17797, (reimpr. 1963), parágrafo 54.
} 
común de ente; porque responde a las determinaciones más generales del ente. El conocimiento de Dios será, además, un conocimiento derivado, ya que la verdadera certeza, la certeza característica de la metafísica como ciencia, se encuentra en los primeros principios, de tal forma que el conocimiento de Dios sólo será posible tras conocer las razones comunes del ser.

Considero que esta interpretación tiene el problema de que la obra de Suárez no es comprendida a partir de su sentido intrínseco, sino desde sus resultados a un siglo vista, en concreto, como el antecedente de las propuestas ya plenamente ontologistas de Wolff y Baumgarten. Creo que estudiar la metafísica de Suárez en sí misma, en su singularidad, exige distanciarse de este enfoque ontologista. Para entender dicha singularidad considero fundamental investigar el lugar que el ens rationis ocupa en la metafísica de Suárez. Por un lado, Suárez se niega introducirlo como un objeto adecuado a la ciencia suprema, por otro, entiende que la metafísica no puede dejar de hacerse cargo de estos entes en tanto que esta ciencia tiene un papel fundamentador respecto del resto de las ciencias. Y es que, como veremos, los entes de razón constituyen piezas fundamentales en el funcionamiento y desarrollo del saber científico.

\section{Rasgos fundamentales del ens rationis}

Partamos de la definición de Suárez da de los entes de razón. Al respecto, afirma que se trata de entes cuyo ser se agota en estar objetivamente en el entendimiento, es decir, cuyo ser extramental no es posible. Obviamente, no todo lo objetivo es ente de razón:

lo que está de este modo objetivamente en el entendimiento, a veces tiene o puede tener en sí un verdadero ser real, según el cual se ofrece como objeto a la razón, y esto no es absoluta y simplemente un verdadero ente de razón, sino real, puesto que este ser concreto es lo que absoluta y esencialmente le conviene, mientras que el ofrecerse a la razón como objeto le es extrínseco y accidental $(54,1,6)$.

Suárez es consciente de la importancia que adquiere la distinción entre el ente real y el ente de razón una vez que ha procedido a definir el ser desde el punto de vista de la essentiam realem, lo que apunta más al ámbito de lo posible que del ser existente $^{10}$. A este respecto, y de acuerdo con lo que ya hemos dicho en la introducción, Suárez inaugura una forma propiamente barroca de entender el ente de razón, distinta de la escolástica tomista tradicional y, por supuesto, de los desarro-

10 "el ente, tomado con valor de nombre, significa lo que tiene esencia real, prescindiendo de la existencia actual, sin excluirla ciertamente o negarla, sino sólo abstrayendo de ella precisivamente" $(2,4,9)$. 
llos que la noción tendrá en la modernidad postbarroca representada nítidamente por Kant.

Efectivamente, si rastreamos lo que Tomás de Aquino dice respecto a los entes de razón por ejemplo en su Summa Theologiae, lo primero que constatamos es que los entes de razón tienen una presencia muy reducida y absolutamente tangencial al objeto de la obra. En segundo lugar, la distinción entre el ser y el no ser (ente de razón) es absoluta, con lo que tal ente resulta absolutamente extraño a la verdad y carece de todo interés científico11.

En cambio, desde la perspectiva del Barroco, aunque se sigue manteniendo que aquello a que refieren los entes de razón, como indica su nombre, no son verdaderos entes, constituyen sin embargo elementos fundamentales para el conocimiento científico. Cosa obvia para los pensadores barrocos en lo que se refiere, como veremos, a las relaciones de universalidad o a ciertos entes matemáticos, como la sucesión numérica. Aunque Suárez obviamente no le da a los entes matemáticos la importancia que tendrán en las filosofías de Descartes o Spinoza, lo cierto es que asienta para el siglo barroco su consideración como entes de razón. En el último apartado del presente trabajo analizaremos con cierto detenimiento el caso de Spinoza, pero la cuestión también valdría para Descartes. A este respecto podemos leer en Los principios de la filosofía: "el número, considerado en general, sin hacer reflexión sobre alguna cosa creada, no es fuera de nuestro pensamiento al igual que cualquiera de la otras ideas generales que, en la escuela, se denominan universales"12.

Vemos entonces cómo aunque el Barroco ha caído en la cuenta de la importancia de los entes de razón para las ciencias, aún tiene problemas para considerarlos como parte integrante de la metafísica, al no haber sido superada la perspectiva existencial. Posteriormente, en la filosofía escolar alemana del siglo XVIII y, sobre todo, en Kant, el giro ontológico de la metafísica será consumado, con lo que la realidad pasará a medirse en orden a la posibilidad lógica y no a la potencia existencial. En consecuencia, el ente de razón se identificará con el imposible lógico y no con el imposible existencial. Desde este punto de vista ya no tendrá sentido llamar "entes de razón" a los entes matemáticos. Así, podemos leer en Kant: "los número [...] no pueden ser contados entre las posibilidades, aunque tampoco por ello hayan de ser considerados imposibles (ens rationis)"13. Resumidamente: en la perspecti-

11 T. de Aquino, Summa Theologiae, C. 16, aa. 3, 5, 7; C. 48, a. 1.

12 R. Descartes, Los principios de la filosofia, Madrid, Alianza, 1995 ( $1^{\text {a }}$ ed. orig. 1644), p. 56 (parte $1^{a}$, pr. 58). Traducción de Guillermo Quintás. Para el estudio comparativo de la distinción de razón en Suárez y Descartes puede consultarte: J. Zubimendi, "La teoría de las distinciones de Suárez y Descartes", Pensamiento, 40 (1984), pp. 179-202.

13 I. Kant, Crítica de la razón pura, Madrid, Alfaguara, 1978 ( $1^{\text {a }}$ ed. orig. 1781), p. 295 (A 290, B 347). Traducción de Pedro Ribas. 
va tomista, el ente de razón, cuyo modelo es la quimera, resulta insignificante desde el punto de vista de la metafísica. En el Barroco, y a partir de Suárez, la quimera pierde fuerza como ente de razón respecto a determinados entes de razón fundamentales para el desarrollo de las ciencias. Entonces la metafísica no puede dejar de hacerse cargo de ellos en tanto que ciencia fundamentadora del resto de las ciencias. En Kant, y una vez que ha sido superada la metafísica entendida en orden a la existencia, no tiene sentido entender las relaciones y negaciones importantes para la ciencia como entes de razón. Ahora el ente de razón paradigmático vuelve a ser la quimera y, con ello, de nuevo pierde interés desde un punto de vista metafísico.

Pero más acá de este excurso histórico y volviendo de nuevo a nuestro tema, en Suárez la distinción entre el ente de razón y el ente real es clara:

ente de razón es aquel que tiene ser objetivamente sólo en el entendimiento, o que es aquel que es pensado por la razón como ente, aun cuando en sí no posea entidad [ens rationis esse illud quod habet esse obiective tantum in intellectu, seu esse id quod a ratione cogitatur ut ens, cum tamen in se entitatem non habeat] $(54,1,6)$.

Respecto a la clasificación de los entes de razón, hay que hacer referencia a tres categorías básicas: negaciones, relaciones de razón y quimeras. Al tratar de aclarar en qué consiste cada uno de ellos, Suárez deja de lado la quimera y se centra en las negaciones. Lo cual, como ya hemos apuntado y como veremos detenidamente más adelante, es altamente significativo. Estas negaciones quedan a su vez divididas en negación propiamente dicha, privación y relación de razón (54,3,3-6). Son relaciones de razón aquellas que son producidas por el mismo entendimiento y, por lo tanto, no tienen realidad extramental. Por su parte, las negaciones son aquellos entes de razón a cuyo través pensamos como real una carencia de forma en un sujeto para la que ni siquiera es acto. Como cuando, por ejemplo, decimos de un topo que es ciego. Por último, las privaciones sirven para pensar como real una carencia de forma en un sujeto para la que sí que es apto. Como cuando por ejemplo decimos de un hombre que es ciego.

Tras esta primera caracterización de los entes de razón es más fácil entender por qué son de extrema importancia para entender el sentido histórico último de la metafísica de Suárez.

En primer lugar, habitualmente, los entes de razón han sido considerados por el suarismo como un agregado a su metafísica, un agregado poco importante y que desempeñan sólo un papel pedagógico o didáctico al permitir entender mejor, por contraposición, qué es un ente real. Por ello el paradigma de ente de razón ha sido, en esta lectura de Suárez y en un intento desesperado por salvarlo de las garras de la modernidad y devolverlo a la vecindad tomista, la quimera ${ }^{14}$.

14 Para hacer justicia a la tradición suarista habría que mencionar aquí el excelente trabajo sobre los 
En segundo lugar, la diferencia entre el ente de razón y el ente real ha sido un elemento que el suarismo anti-moderno ha utilizado para rechazar las interpretaciones ontológicas del filósofo granadino. Resumidamente la cuestión se presentaría de la siguiente forma ${ }^{15}$ : si entendemos la esencia real haciendo referencia sólo al ámbito de lo posible objetivo, ¿cómo distinguirla de la mera ficción, de lo posible meramente fingido? Para distinguir el ente real del ente de razón es necesario acudir al ámbito de la causación. El ente real no es comprensible en Suárez si no se ve desde la referencia a las causas. Desde esta perspectiva, el ente de razón nos estaría exigiendo distinguir el ente meramente posible del posible efectivo. Algo no contradictorio podría ser fabricado por la mente y que, al mismo tiempo, no tuviera aptitud para existir.

El problema de esta interpretación es que no tiene en cuenta que Suárez ha dejado bien claro que en ningún caso el ente de razón puede entenderse como subsumido en lo posible. Precisamente, el ente de razón no puede ser incluido bajo el concepto común de ente, porque no es ser, en el sentido de que no es algo posible, sino meramente pensado o fingido:

Por eso una descripción común, cual es la que puede darse sobre el concepto común de ente, a saber lo que tiene ser, no le conviene en realidad a los entes de razón, motivo por el que tampoco se puede decir que posean esencia, ya que la esencia, considerada absolutamente, expresa referencia al ser o capacidad de él; en cambio, el ente de razón es tal "que le repugna el ser" [Unde communis descriptio, qualis dari potest de communi conteptu entis, scilicet, id quod habet esse, revera non convenit entibus rationis, et ideo nec dici possunt habere essentiam, quia essentia, simpliciter dicta, dicit habitudinem ad esse seu capacitatem eius; ens autem rationis tale est, ut ei repugnet esse] $(54,1,10)$.

Aquí, "que le repugna el ser" significa que no es posible; como dice líneas más abajo "en sí mismo no es nada [in se nihil est]". Pensemos que la nada ya no es entendida desde el horizonte de la causalidad, sino del de lo no-posible. Solamente en referencia a la imaginación podemos fingir entes de razón posibles, "como cuando finge un monte de oro, el cual no existe, aunque sea posible" $(54,2,18)$. Pero cuidado, el ente real en orden a la esencia real nunca puede pensarse en orden a la imaginación, sino a la razón, así que no hay dificultad en distinguir el posible imaginativo del posible racional. De hecho, sólo analógicamente podemos llamar a este ente de imaginación ente de razón, por la proporción que guarda con él, pero el verdadero ente de razón nunca es un posible.

entes de razón en Suárez de Juan F. Yela, quien ya en 1948 cayó en la cuenta de la importancia que adquirían respecto a lo que había sido su tratamiento escolástico anterior, donde eran prácticamente despreciados. Cf. J. F. Yela, "El ente de razón en Suárez", Pensamiento, 4 (1948), pp. 271-303.

15 Esta posición ha sido mantenida por Luís Martínez Gómez en "Suárez y las raíces espirituales del barroco español”, Cuadernos Salmantinos de Filosofia, 16 (1989), pp. 127-146. 
Respecto al primer asunto que hemos destacado, la importancia que las negaciones adquieren, como entes de razón, respecto a las quimeras, es muestra de la enorme importancia, ya apuntada, que los entes de razón tienen en la filosofía de Suárez y, a partir de él, en la filosofía moderna. Las negaciones no son meros caprichos, como los que producen las quimeras (Suárez hace referencia a la hora de explicar cómo se producen las quimeras, a la fecundidad del entendimiento, que, partiendo de entes verdaderos, puede crear otros ficticios e imposibles). Entender por qué son tales entes de razón realmente importantes, es entender la razón profunda de su tratamiento en el ámbito de la metafísica, más allá de cuestiones meramente didácticas que remarcarían el carácter existencial del ente real.

Nos vemos así lanzados a la pregunta realmente importante para el presente ensayo: ¿cuál es la razón por la que nuestro entendimiento produce tales entes de razón? Tenemos la fortuna de que Suárez ha respondido nítidamente a ello $(54,1,8)$.

En lo que se refiere a las negaciones propiamente dichas, nuestro entendimiento no puede hacerse cargo de ellas sin pensarlas a modo de entes, ya que, nos dice Suárez, la noción de ente está a la base de nuestra capacidad intelectiva. En lo que se refiere a las relaciones de razón, nuestro entendimiento no puede aprehender las cosas tal como son en sí en una aprehensión intelectual pura, sino que necesita buscar rutas, elegir caminos, y por ello establece comparaciones, relaciones de razón, que en la misma realidad no se dan.

En el asunto de los entes de razón es donde Suárez se mueve de una manera más confusa en las aguas pantanosas que separan el medioevo de la modernidad. Suárez aún se niega a considerar lo meramente objetivo como lo real, así el ente de razón se contrapone al ente real, y en la primera disputación es tajante, siguiendo a la tradición a la hora de dejar los entes de razón fuera del objeto de la metafísica.

La primera razón que Suárez encuentra para rechazar el carácter real de los entes de razón, está en la tradición. El asunto aparece justo al principio de las Disputationes, a propósito de la discusión en torno al objeto de la metafísica. Suárez, como buen escolástico, es tajante al respecto: los entes de razón no pueden ser incluidos en la metafísica porque incluirlos va "manifiestamente en contra de lo que afirma Aristóteles en el libro VI de la Metafisica" $(1,1,5)$, donde el Filósofo, no duda en excluirlos de la consideración directa de esta ciencia.

En segundo lugar, Suárez, en estos primeros pasos de sus Disputationes, parece dar la razón a suaristas contemporáneos como Luis Martínez ${ }^{16}$ en la afirmación de que el estudio de los entes de razón tiene sólo un fin pedagógico:

si esta ciencia dedica de alguna forma su atención a los entes de razón, no es por sí mismos, sino por cierta proporcionalidad que tiene con los entes reales, y esto con el fin de distinguirlos de ellos y dar a entender mejor y más claramente qué es lo que tiene entidad y realidad en los entes y qué es lo que no tiene sino apariencia $(1,1,6)$.

16 Ibíd. p. 129 y ss. 
Pero al avanzar en la obra, conforme esta va apropiándose del terreno ontológico y fundamentador del resto de ciencias, y a medida que Suárez se deja invadir por el espíritu de la totalidad que va diluyendo los esquemas dualistas heredados por la tradición aristotélico-tomista ${ }^{17}$, ya no puede detenerse ante los entes de razón. Siente la necesidad de sacarlos de la dialéctica, para traerlos a la metafísica. Así y finalmente, en espíritu claramente Barroco, encontramos en la disputatio sobre los entes de razón, la superación de un nuevo dualismo, el último que era preciso superar, mostrándose al final triunfante la filosofía en su máxima unidad, la unidad que le propone la metafísica y su noción de ente que todo lo subsume: lo fundamentante y lo fundamentado, lo infinito y lo finito, lo espiritual y lo material, lo existente y lo representado. La metafísica como el máximo pliegue del que todas las demás ciencias son expresión.

En Suárez, realista, esto no puede ocurrir sin tensiones, pero cuando la modernidad haga coincidir lo real con lo representado, los entes de razón o, mejor dicho, los entes ideales, entrarán con todo derecho en la metafísica. Entonces, el ente ideal será de importancia vital para una época que conoce su capacidad de creación y dominio. Frente a la tendencia del espíritu medieval de dejar el momento creativo en manos de Dios, el hombre moderno (o, más exactamente, a partir del hombre renacentista, como muestra el humanismo de Pico de la Mirándola), basa su concepción de la vida, como tiempo, en el acto creativo mismo, que define su propia esencia humana. El ente ideal, la idea, funda el ser en el pensamiento moderno. Si el punto de partida ya no es el mundo de las cosas exteriores, sino el mundo de la conciencia (y dado que el yo es en lo fundamental un ente creador de ideas, representativo), lo fundamental para la metafísica pasará a ser la representación del mundo y no el mundo mismo (que acabará convirtiéndose en un sinsentido).

De Suárez no podemos decir que sea moderno consumado si entendemos por tal el tomar como punto de partida del filosofar la libertad o creatividad humana (en esto es incluso prerrenacentista). Obviamente en él el idealismo no ha sido aún consumado y el sujeto continúa en un segundo plano.

Con todo lo dicho hasta ahora, es claro que el ente de razón, es decir, el ente meramente objetivo, no tiene su lugar en la metafísica por constituir la esencia misma del ser (posición que consumará el ontologismo puro), sino por un motivo mucho más modesto, pero, al mismo tiempo, no menos importante: el ente de razón ha de ser tratado por la metafísica por el carácter fundamentador de esta disciplina respecto a todas las demás.

17 Cf. L. Martínez Gómez, "Para una evaluación histórica de Francisco Suárez", Cuadernos Salmantinos de Filosofia, 7 (1980), pp. 5-25. También puede verse mi trabajo: "La mitigación de los dualismos metafísicos en Francisco Suárez. Para entender la transformación moderna de la metafísica”, en J. M. Navarro y V. Sanfélix, IV Congreso internacional de la SAF, Madrid, Sociedad Académica de Filosofía, 2009, pp. 193-201. 
En este momento Suárez se ve en la obligación de matizar su posición inicial, la que encontrábamos en la primera disputatio, respecto a los entes de razón:

Aunque en la disputación primera de esta obra hayamos dicho que el ente de razón no está comprendido bajo el objeto directo y propio de la metafísica, y por ello había sido excluido de este tratado por el propio Filósofo en el libro VI de su Metafísica, sin embargo, pienso que el complemento de esta disciplina y la tarea del metafísico exige explicar los puntos generales y comunes a los entes de razón $(54,1,1)$.

La determinación negativa del ente de razón como carente de realidad, no le hace perder su valor. Los entes de razón (ya sean negaciones, privaciones o relaciones, y a diferencia de la mera quimera) no son un capricho de la razón o una excrecencia suya, sino elementos esenciales en nuestro afán de conocimiento del mundo. De hecho, en el pasaje que leíamos antes, en el que Suárez explicaba "por qué se han ideado los entes de razón", la diferencia entre negaciones, privaciones y relaciones de razón, por un lado, y las mera ficciones, por el otro, es clara: las primeras, recordemos el texto,

de alguna manera se fundan en las cosas, o se ordenan al conocimiento de algo que se puede afirmar con verdad de las cosas mismas [fundantur aliquo modo in rebus, vel ordinantur ad cognoscendum aliquid quod vere de rebus ipsis dici potest $(54,1,8)$.

La ciencia, en su sentido clásico, sería inviable sin negaciones y relaciones de razón, y, por supuesto, aunque Suárez no fuera aún consciente de ello, la ciencia moderna, la mecánica, no podría haber llegado a ser sin la intervención de unos entes de razón muy especiales: los matemáticos.

Llegados a este punto, en el análisis que a partir de ahora voy a proponer de los entes de razón en la filosofía de Suárez, voy a responder sucesivamente a tres cuestiones, de las que avanzo ya una breve respuesta.

En primer lugar, ¿por qué trata la metafísica los entes de razón? Ya hemos respondido en parte a esta cuestión. Insistiremos en el carácter fundamentador de la metafísica y en el papel que desempeña con vistas a asegurar la unidad de las ciencias. En todo ello, obviamente, los entes de razón cumplen funciones de extrema importancia.

En segundo lugar, ¿cuál es el valor científico de los entes de razón? La respuesta a esta pregunta pasa por la manera que Suárez tiene de solventar el problema de los universales una vez que mantiene, con el nominalismo, que sólo existen realidades singulares. A este respecto, los entes de razón constituyen un eslabón esencial entre una realidad de carácter singular y una ciencia que necesariamente, para ser tal, ha de serlo de lo universal. 
Por último, y además como una interesante curiosidad que se deriva del sentido de la metafísica en Suárez, constatando ya en él cierta importancia adquirida por los entes de razón, ¿por qué, aún así, no es posible una física matemática?

\section{La unidad de las ciencias}

Sabemos ya que si la metafísica se ocupa de los entes de razón, y contra lo que Suárez dijera al comienzo de las Disputationes, no lo hace por una cuestión meramente pedagógica, sino porque el conocimiento de dichos entes y su uso parece esencial para el desarrollo de las ciencias, y, por lo demás, sólo pueden ser estudiados por la metafísica:

En efecto, el conocimiento y ciencia de los mismos es necesario para las disciplinas humanas, ya que sin ellos apenas podemos hablar ni en metafísica, ni tampoco en filosofía (natural), ni mucho menos en lógica, y -lo que aún es más importante- tampoco en teología. Y esta tarea no puede corresponder a ningún otro más que al metafísico $(54,1,1)$.

La razón por la cual, aun siendo entes necesarios para todas las ciencias, no pueden ser abordados por éstas, se encuentra en que al no ser verdaderos entes, no pueden ser objeto de conocimiento de ninguna ciencia particular:

en mi parecer no pertenecen por sí y primariamente a ninguna ciencia, ya que no siendo entes, sino privaciones de entidad, no son cognoscibles por sí más que como cosas que se apartan de la verdadera razón de ente $(1,1,7)$.

En este texto, todavía al comienzo de las Disputatio, el desprecio del ente de razón es claro, pero gracias a él, dicho ente queda al margen de las ciencias particulares y la necesidad de su tratamiento no responde, por tanto, a la necesidad de conocerlos en sí mismo, sino a la necesidad de reglar y fundar el conocimiento científico:

Porque, en primer lugar, por no ser los entes de razón entes verdaderos, sino como sombras de entes, no son inteligibles por sí, sino en virtud de cierta analogía y conexión con los verdaderos entes, y, en consecuencia, tampoco son por sí objeto de conocimiento, ni existe ciencia alguna que haya sido instituida esencial y primariamente sólo para conocerlos (...). Por consiguiente, ningún artífice o ninguna ciencia pretende esencial y primariamente el conocimiento de los entes de razón, sino que este conocimiento debe exponerse en cuanto está vinculado con el conocimiento de algún ente real; éste es el sentido en el que el físico trata de la privación, la cual, juntamente con la materia, está unida con la forma; y trata del vacío por comparación con el lugar, etc. $(54,1,1)$. 
Su conocimiento, por tanto, es necesario en un intento de fundar las ciencias. No son objeto de las ciencias mismas, sino del saber reflexivo que funde dichas ciencias. Y dado que la metafísica es la ciencia fundamentadora respecto a los demás saberes, es esencial que se haga cargo de dichos entes de razón. Recordemos a este respecto lo que Suárez nos decía en el Proemio de las Disputationes:

entre todas las ciencias naturales, aquella que ocupa el primer lugar y obtuvo el nombre de filosofía primera, es la que principalmente ayuda a la teología sobrenatural; ya porque es la que más se acerca al conocimiento de las cosas divinas, ya también porque es ella precisamente la que explica y confirma los principios naturales que abarcan todas las cosas y que, en cierto modo, sustentan y mantienen toda ciencia.

Suárez no puede ocultar cierto placer en incluir los entes de razón en la ciencia de lo transcendental, en consumar la eliminación del último dualismo en la ciencia omniabarcante de lo trascendental, haciendo referencia a su cuasi-trascendentalidad:

De este modo, pues, es propio de la metafísica tratar del ente de razón en cuanto tal, y de la razón común, propiedades y divisiones del mismo, puesto que estas razones son a su manera cuasi trascendentales [quai hae rationes suo modo sunt quasi transcendentales] y no se pueden entender más que por comparación con las verdaderas y reales razones de entes, bien las trascendentales, bien las hasta tal punto comunes, que sean propiamente metafísicas, ya que lo que es ficticio o aparente debe entenderse por comparación con lo que verdaderamente es (54, introducción).

En conclusión, la metafísica tiene como una de sus funciones aclarar en qué consisten los entes de razón con el objetivo de fundamentar el objeto de las ciencias particulares. La metafísica aparece así como el pliegue de pliegues, la unidad máxima del saber y la realidad representada por dicho saber, a partir de la que todo puede ser investigado con sólo tirar del pliegue mismo. Un tirar que, en tanto situado en el ámbito del entendimiento, no tiene fin:

El exponer más puntos sobre estas relaciones es tarea de las ciencias particulares, ya que semejantes relaciones pueden prácticamente multiplicarse hasta el infinito mediante ficciones y reflexiones del entendimiento, $\mathrm{y}$, en consecuencia, tanto de ellas como de toda esta ciencia baste con lo que hemos dicho, que deseamos redunde en gloria de Dios $(54,6,11)$.

Afirmaba, en las primeras páginas de este trabajo, que la metafísica de Suárez tiene una finalidad profundamente barroco-jesuítica. Al respecto, podríamos decir que sólo ahora, cuando Suárez ha conseguido integrar el ente de razón en la metafísica, se ha plasmado el ideal ignaciano de cerrar en una mirada plenamente natural, 
donde natural quiere decir racional, la revelación divina. El principio de no-contradicción aparece como pliegue en su máxima densidad, y en cuya desenvoltura surge el todo de lo real en su representación para gloria de Dios. Dios permanece como algo extrínseco al sistema, pero sólo por cierto tiempo, al final él mismo será naturalizado, ya que la tendencia a la eliminación de dualidades no tendrá fin hasta subsumir la misma trascendencia. Cuando dicha trascendencia sea absorbida por el sistema, la referencia externa de la objetividad dejará de tener sentido. Lo racional coincidirá con lo real y lo real con lo racional. Será el momento de la consumación antropológica de la filosofía.

Aterrizando de nuevo en nuestro propósito actual, más acá de estas consideraciones generales sobre el sentido de la filosofía moderna, Suárez ha insistido en la unidad y ordenación de las ciencias en la Disputatio XLIV, en la que trata de los hábitos, especie propia del modo de la cualidad.

En su acepción más general, los hábitos son cualidades que se añaden a nuestras potencias por el uso y la costumbre de los actos con el fin de facilitar nuestras operaciones. Suárez nos dice en pocas palabras de qué clase pueden ser:

distinguimos dos géneros de cualidades, que se añaden a nuestras potencias para ayudar a éstas en orden a sus operaciones; unas sirven para unir el objeto a la potencia, y de ellas se dice que son requeridas por parte del objeto, y sólo resultan necesarias en las potencias cognoscitivas; hay otras que se añaden a la potencia para aumentar el poder de ésta en el obrar, de donde les viene el conferir facilidad en la operación, lo cual consta con mayor evidencia en la voluntad, porque ésta no necesita cualidad por parte del objeto, $y$, sin embargo, con el uso adquiere facilidad en el obrar $(44,1,3)$.

Obviamente, ahora nos interesan los hábitos que perfeccionan las cualidades cognoscitivas, es decir, los hábitos científicos.

La cuestión es compleja e intentar presentarla aquí en todos sus matices nos desviaría demasiado de la materia. Aún así en ella se abordan asuntos de gran interés respecto al tema de los entes de razón y su importancia para la ciencia. Intentaré por ello exponerla de la forma más simple posible sin traicionar el espíritu de lo que Suárez nos quiere decir. Para ello, y aún a riesgo de caer en una citación excesiva, dará la palabra al propio Suárez.

Para entender cabalmente la cuestión es preciso partir de la distinción que establece Suárez entre el conocimiento evidente y el que no lo es. Al respecto nos dice lo siguiente:

Se da entre ellos la diferencia de que los hábitos evidentes muestran la verdad de la cosa en sí misma, ya sea por los propios términos, ya sea por algún medio; en cambio, los inevidentes no muestran la verdad en sí, sino que por medios extrínsecos inducen al entendimiento a que asientan, aun cuando no intuya la verdad $(44,11,45)$. 
Y en el parágrafo siguiente nos hace ver que "un hábito inevidente puede inclinar a varias materias con mayor facilidad que uno evidente" $(44,11,46)$. Ello se debe a que una vez que alcanzamos una evidencia, esta no puede aplicarse sin más a otras proposiciones, porque la razón de asentir es intrínseca; pero cuando la razón de asentir es extrínseca, como ocurre en la opinión, sí que se puede aplicar de manera uniforme a distintas materias.

Una cuestión muy importante, que se deriva de esta conclusión, respecto al conocimiento científico (que es, obviamente, el evidente) es la de si es posible hablar de una unidad en el mismo y, en caso afirmativo, en qué sentido podemos hacerlo. Porque la primera solución a la que estamos inclinados es, al respecto, algo pesimista:

avanzando más a los hábitos de la ciencia, no parece posible que un mismo hábito indivisible sea suficiente para asentir a diversas conclusiones, no sólo cuando se demuestra acerca de cosas diversas de especie o en esencia, sino también cuando se demuestran acerca de la misma ciencia $(44,11,51)$.

Y un poco más adelante: "un hábito indivisible, adquirido con anterioridad, no puede bastar esencialmente y sin adición real, para dar al entendimiento prontitud para asentir a esa verdad distinta" $(44,11,52)$.

Dicho de otra forma, parece que para que el hábito de la ciencia, en sí indivisible, se aplique a otras materias, es necesario que se produzca un aumento extensivo del mismo. Pero, ¿cómo podría producirse tal aumento? Aún la extensión de la cita, lo mejor es dejar a Suárez expresarse en este punto:

la ciencia especulativa se detiene en el conocimiento de los universales, y por eso, en cuanto de ella depende, no aplica la misma verdad formal a muchas cosas materialmente diversas, sino que siempre se extiende por adición de una nueva verdad, que en su universalidad es formalmente diversa de otra, por lo que requiere no sólo un asentimiento formalmente diverso, sino también un real aumento o extensión del hábito que inclina a ella. En consecuencia, si el hombre aplica una conclusión científica demostrada universalmente a los particulares contenidos en ella, en este sentido un mismo hábito indivisible de la ciencia inclinará igual y suficientemente a todas las verdades particulares contenidas en la universal; por ejemplo, si, habiendo adquirido la ciencia de esta conclusión: el hombre es risible, se infiere en sentido expositivo: Pedro es hombre, luego Pedro es risible, y así en los demás casos, para todos los asentimientos a esas conclusiones particulares bastará el mismo hábito indivisible, porque esas verdades difieren materialmente más bien que formalmente, y porque todas esas proposiciones particulares están contenidas de manera confusa en la universal, y por esto sólo es necesario que se añadan las especies o la aplicación de ellas a los singulares $(44,11,54)$. 
Dado que las ciencias se distingue por su diverso grado de abstracción, la ordenación de las mismas está prácticamente servida: aquellas cuyo objeto goce de más universalidad subsumirán a aquellas cuyo objeto sea menos universal:

esta unidad del objeto en las ciencias debe tomarse del grado de abstracción; y así suelen distinguirse tres clases de ciencia por la triple abstracción de la materia: o solamente de la individual, y no por completo de la materia sensible, o de la materia sensible y no de la inteligible, o de todas $(44,11,68)^{18}$.

A su vez, el despliegue del universal más abstracto al menor irá produciendo el despliegue de cada ciencia y sus particularidades. Está asegurada así la unidad de la ciencia, unidad en pluralidad de despliegue, aunque para ello sea necesario hablar de distintos hábitos y de la coordinación y subordinación entre ellos. Se puede explicar por qué la metafísica es la única ciencia que engloba todos los saberes sin necesidad de pensar que le corresponde un hábito simple, sino que es suficiente con partir de "una unidad compuesta por colección y subordinación de varias cualidades que, con respecto a ese hábito íntegro, se consideran hábitos parciales" $(44,11,55)$. Lo que explica que el descender de la metafísica, ciencia integradora, a otros saberes, exija hábitos distintos.

Es interesante constatar cómo Suárez toma distancia de Tomás de Aquino en este tema:

Santo Tomás piensa que ese hábito así aumentado y perfeccionado siempre sigue siendo una cualidad simple, cosa que yo admitiría con gusto, si entendiese, o bien que esa extensión puede producirse por aumento puramente intensivo, o bien que en el aumento extensivo puede intervenir una unión real y esencial entre las cualidades parciales. Mas, no siendo posible entender ninguna de estas dos cosas, como he demostrado, por eso afirmo que esa ciencia es una, pero no un hábito simple. Y no prueba más la razón que Santo Tomás añade, a saber, que las conclusiones y demostraciones de una ciencia están ordenadas y una se deriva de otra. Porque, a lo sumo, de ahí se concluye la subordinación de dichas cualidades y cierta unidad de toda la ciencia, pero no su perfecta simplicidad o su composición per se $(44,11,56)$.

Ahora entendemos la razón por la cual Suárez se ha visto forzado a llevar a cabo una nueva sistematización de la metafísica, a romper con el orden de tratamiento impuesto a partir del siglo XII en la Escolástica en la forma de comentario a la

\footnotetext{
18 No olvidemos nunca el realismo suareciano en su manera de pensar la abstracción: "la abstracción, si se toma por nuestra parte, no puede constituir el objeto formal, porque esta abstracción, con respecto a las cosas, no pasa de ser una denominación extrínseca; y ya hemos demostrado que el objeto formal debe presuponerse por parte de las cosas mismas escibles. En cambio, si se considera fundamentalmente por parte de las cosas mismas, entonces con esta abstracción sólo se significa el orden o grado de cada una de las esencias más o menos inmateriales" $(44,11,68)$.
} 
Metafisica de Aristóteles: el orden y la unidad de las ciencias no está dado, sino que ha de ser producido, porque el hábito de la ciencia no es una cualidad simple. Vemos cómo con ello el problema del método comienza a ocupar un lugar relevante en el ámbito científico.

Nos queda saber por qué el problema de la unidad y organización de las ciencias resulta tan importante para Suárez. Al respecto nos dice que "todas las ciencias parecen tener entre sí tal conexión, que ninguna puede enseñarse de manera perfecta sin las otras" $(44,11,59)$. Y así resulta, de forma muy parecida al proceder que Descartes seguirá en sus Principios de filosofia, que "las ciencias prácticas dependen mucho de las especulativas, y las cosas morales de las físicas, y las artificiales de las naturales y matemáticas" $(44,11,59)$.

La cuestión no era baladí, porque el espíritu nominalista, tan impuesto por esta época en el ámbito escolástico, venía a sostener que había tantas ciencias como hábitos simples, resultado, además, imposible encontrar la unidad entre ellas; por otro lado, dado que había distintas dialécticas, teologías, físicas, etc., ocurría que incluso dentro de una misma disciplina era posible hacer referencia a diversas ciencias. La situación había llegado, con ello, a un momento realmente caótico $(44,11,60)$.

Por lo demás, se puede constatar cómo el artificio barroco no puede dejar de aparecer en esta manera de pensar la unidad de las ciencias: "Y estimo que hay que filosofar así acerca de las demás. Ello aparecerá menos difícil para quien considere que esta unidad de la ciencia no es exacta y perfecta, sino cuasi artificial" $(44,11,69)$. Suárez es así consciente de todos los problemas que conlleva esta manera de entender la unidad de la ciencia.

\section{El valor científico de los entes de razón}

Ya he hecho referencia anteriormente a la distinción que Suárez establece, dentro de los entes de razón, entre las quimeras y las negaciones en general (recordemos que incluyen negaciones propiamente dichas, relaciones de razón y privaciones). Ahora es el momento de explicar el sentido último de esta tajante distinción.

En primer lugar, aunque todos son entes de razón y por lo tanto en ningún caso tienen realidad extramental, los segundos tienen fundamento en las cosas, mientras que los primeros no la tienen en absoluto:

hay algunos entes de razón que tienen fundamento en las cosas, aunque reciban su complemento de la razón [habent in rebus fundamentum, licet a ratione habeant complementum], como la razón de universal o la de género y otras semejantes, y de este tipo son también las negaciones y las privaciones [et huiusmodi etiam sunt negationes et privationes] (...). Por el contrario, hay otros entes de razón completamente fingidos por el entendimiento sin fundamento en la realidad, por ejemplo, la quimera $(54,4,2)$. 
En segundo lugar, si nos fijamos en los ejemplos de relaciones de razón que Suárez utiliza para remarcar la distancia entre la quimera y el resto de entes de razón, como son las razones de universal o género, entendemos enseguida que los entes de razón con fundamento en la realidad desempeñan un papel fundamental en el conocimiento científico:

tal ente de razón sirve de alguna manera para las ciencias y para los conocimientos de las cosas tal como pueden darse en los hombres, pudiendo de esta suerte ser objeto de ciencia y de enseñanza [huiusmodi ens rationis aliquo modo deservit ad scientias et cognitiones rerum prout in hominibus esse possunt, et ita potest sub scientiam et doctrinam cadere]; por tanto, dado que se trata de una división científica, se propone legítimamente sólo para ese ente de razón, ya que la otra clase de ente de razón meramente ficticio es completamente accidental y puede multiplicarse hasta el infinito $(54,4,2)$.

Sin duda, uno de los temas mas espinosos para los estudiosos de la filosofía de Suárez es el de determinar el papel gnoseológico y ontológico del universal ${ }^{19}$ en su filosofía si partimos de que para Suárez hay sólo un tipo de verdadera unidad en el universo: la unidad individual $(5,1,4$ y 5). Pues bien, creo que la posición de Suárez, respecto a qué entiende por el universal, y su rechazo de la posición nominalista (sobre todo en sus posiciones más radicales, como puede ser la de Roscelino, para quien los universales no son más que expresiones, voces, particulares), se explica desde esta concepción del ente de razón como necesario para el saber científico. Es una tesis conflictiva, pero señalada por él mismo en la disputatio LIV, y desarrollada ambiguamente en la VI. Al respecto, en la citada disputatio LIV podemos leer lo siguiente:

\footnotetext{
19 Soy consciente de que el problema de los universales en Suárez requeriría un mayor tratamiento y medir la interpretación que voy a proponer aquí con las que han llevado a cabo otros estudiosos de la obra de Suárez, pero dados los límites de extensión exigidos por el formato de un artículo, no puedo más que aplazar la cuestión a un trabajo posterior. Para hacerse cargo del asunto en toda su problematicidad, pueden consultarse, entre otros, los siguientes trabajos: E. Åkerlund, "Suárez on Forms, Universals and Understanding", Studia Neoaristotelica, 6/2 (2009), pp. 159-182; M. Álvarez, "Sobre el concepto de individuo en F. Suárez", en Francisco Suárez (1548-1617), tradição e modernidade, en A. Cardoso, Lisboa, Edições Colibri - Centro de filosofia da Universidade de Lisboa, 1999, pp, 45-64; J. E. Gracia, "Suárez y la individualidad", Cuadernos salmantinos de filosofia, 10 (1983), pp. 157-182; J. E. Gracia, "Suárez and Metaphysical Mentalism: The Last Visit", American Catholic Philosophical Quarterly, 67 (1991), pp. 349-354; J. E.Gracia, Individuation in Scholasticism. The Later Middle Ages and the Counter Reformation 1150-1650, New York, S.U.N.Y. Press, 1994 ; S. Menn, "Suárez, Nominalism and Modes", en K. White, Hispanic Philosophy in the Age of Discovery, Washington, D.C., The Catholic University of America Press, 1997, pp. 201-225; C. G. Noreña, "Ockham and Suárez on the Ontological Status of Universal Concepts", The New Scholasticism, 45/3 (1981), pp. 348-362 ; T. Rinaldi, Francisco Suarez. Cognitio singularis materialis : De Anima, Bari, Levante, 1998; J. B. South, "Singular and Universal in Suarez's Account of Cognition", Review of Metaphysics, 55/4 (2002), pp. 785-823.
} 
nuestro entendimiento no concibe suficientemente a algo como término de la relación de otra cosa con él, sin que lo conciba inmediatamente como algo correlativo, y entonces en la cosa así denominada se concibe algo a modo de un ente relativo, y por no tratarse de un ente real ni de una relación real, resulta consecuentemente que se trata de un ente de razón. Y éste es el modo como explicamos la cuestión antes, disp. VI, sec. 6 y 7, y lo que dijimos allí sobre la elaboración del universal en cuanto tal puede aplicarse aquí a las demás relaciones de razón $(54,2,15)$.

Creo que la posición de Suárez respecto al problema de los universales ha de ser catalogada como un nominalismo de corte conceptista, suficiente para asegurar la validez de la ciencia, en tanto que discurso universal, en su aplicación a los individuos particulares. A tal respecto, es suficiente con que por realidad universal entendamos una denominación extrínseca con fundamento remoto en la cosa:

Sin embargo, estas relaciones no son fingidas gratuitamente, sino tomando algún fundamento por parte de la cosa, como es o la conveniencia real en que se funda la abstracción del universal, la cual luego deriva en género, en especie, etc., según que la conveniencia sea mayor o menor [Non tamen sunt hae relationes gratis conflictae, sed sumpto aliquo fundamento ex re, qualis est aut realis convenientia in qua fundatur abstractio universalis, quae etiam variatur in genus, speciem, etc., ex eo quod convenientia maior est vel minor] $(54,6,9)$.

Obviamente, a lo que se opone Suárez es a un nominalismo extremo (como el que efectúa Roscelino) que, simplemente, elimine tal fundamento remoto. Dicho de otro modo, hay en Suárez una atribución de las intenciones lógicas a las cosas, y no sólo a las voces, pero de una forma tan extrínseca que no podemos dejar de hablar de relaciones de razón.

Esta interpretación del problema de los universales en la obra de Suárez choca con el rechazo explícito por parte del propio autor de que su solución de la cuestión pueda ser entendida en algún sentido como nominalista. Pero hay que tener en cuenta que lo que Suárez entiende por nominalismo va en la línea del radicalismo de Roscelino, que invalida cualquier forma de conocimiento universal, es decir, de conocimiento científico. Si consideramos nominalista la posición de, por ejemplo, Abelardo, quien presenta un nominalismo de carácter conceptualista, Suárez podría ser también considerado nominalista.

Sin duda, en los esfuerzos por parte de Suárez para que su posición no fuera catalogada como nominalista intervienen dos aspectos. En primer lugar, su clara intención por mostrar que se aleja lo menos posible de la solución que Tomás de Aquino diera a los problemas metafísicos (aunque en este caso, dado que Suárez considera que sólo la unidad individual es unidad real, la distancia respecto de Tomás de Aquino es inevitable). En segundo lugar, el peligro, ya constatado por 
Escoto, de caer desde el nominalismo en una crítica escéptica de la objetividad científica.

Pero lo cierto es que Suárez consigue hacerse cargo de las tesis básicas sobre la realidad del nominalismo y al mismo tiempo desarrollar un cuadro conceptual que le permita asegurar la universalidad propia del conocimiento científico. Es cierto, con el nominalismo, que la unidad formal no es realmente distinta de la individualidad ontológica, pero esto no significa que sea una unidad simplemente inventada, ya que, como dice Suárez,

el ser conviene intrínseca y esencialmente no sólo a las cosas singulares, sino también a las naturalezas que son concebidas por nosotros de modo universal, ya que ni son nada absoluto, ni son esencialmente pluralidad de entes individuales, ya que no incluyen las singularidades propias o principios individuantes, sino sólo los principios esenciales; por consiguiente, cada naturaleza de suyo ni es pluralidad de entes ni es pura nada, sino que es un ente real $(6,1,6)$.

Para Courtine20, la tesis de la no-distinción real entre individuo y unidad formal, y la afirmación de que la unidad formal constituye un ente real, sólo resultan compatibles a través del desdoblamiento del concepto de realidad: si por un lado lo real es lo existente-concreto, por otro, aunque las esencias no existen en la realidad antes de la operación mental, no puede decirse que sean meras ficciones:

no las fingimos con la mente, sino que más bien las aprehendemos y conocemos que existen en las cosas, y de dichas naturalezas así entendidas proponemos definiciones, elaboramos pruebas e inquirimos su ciencia $(6,2,1)$.

Con Occam, "la universalidad no se da en las cosas, sino que les conviene en cuanto están objetivamente en el entendimiento", pero, contra Occam "aunque la denominación de universalidad se aplique a las cosas en función de los conceptos, sin embargo, las cosas a las que denominamos así son reales y existen en el mundo real" $(6,2,1)$.

A mi juicio, la interpretación que hace Courtine en este punto resulta bastante problemática. Realmente tal interpretación cuenta con el apoyo de que el mismo Suárez ha considerado que las naturalezas constituyen entes reales; sin embargo, no podemos olvidar que tras tal afirmación encontramos una identificación real entre la unidad de la naturaleza y la unidad del individuo. Sólo se pueden distinguir conceptualmente. La tesis objetivista y ontologista de Courtine cuenta con el inconveniente de que Suárez ha dejado los entes de razón fuera del objeto de la metafísica, porque tras ellos no encontramos esencias reales, al mismo tiempo que, como

20 J.F. Courtine, Suarez et le système de la métaphysique, op. cit., pp. 186-187. 
hemos visto, establece una tajante distinción, en orden a la objetividad entre la quimera y el resto de entes de razón. A mi juicio, la estrategia con la que Suárez soluciona el problema de los universales no implica aún un concepto de realidad diferente a la extramentalidad, sino el rescate objetivo de los entes de razón. Explicar esto exigirá rodeos y algunas repeticiones.

Al enfrentar el trascendental "unidad", Suárez ha comenzado por establecer en la disputación $\mathrm{V}$ que todo lo que existe es singular e individual y a continuación ha dicho que "se dice común o universal aquello que según una razón única se comunica a muchos, o se encuentra en muchos; $y$, en cambio, se llama numéricamente uno, o singular e individual aquello que de tal forma es un ente que según aquella razón de ente por la que se dice uno, no es comunicable a muchos" $(5,1,2)$. Desde aquí, cualquier atribución de realidad al universal está claramente prohibida. Pero al mismo tiempo, como ya sabemos, Suárez quiere evitar a toda costa que esta afirmación pudiera hacerle caer en una posición teórica escéptica que pusiera en cuestión la validez científica. Esto significa que aún cuando el universal no sea en sí mismo real, ha de tener algún referente real.

A este respecto, sostiene que "además de la indivisión numérica individual, llamada material, existe en la cosas una indivisión esencial o formal" $(6,1,1)$. Parece estar apuntando a la naturaleza común de Escoto, pero inmediatamente se opone a él: primero, al negar la distinción real entre ambas formas de indivisión; y, segundo, al negar que se trata de una misma naturaleza que se multiplique en los individuos $(6,1,2)$. La indivisión material y la formal solo se distinguen racionalmente, y hay tantas unidades formales como individuos. Con ello resulta que la naturaleza común sólo puede mantenerse como una relación de razón basada en la conveniencia o semejanza. La unidad formal se identifica con la esencia, y se sostiene que dos individuos, Pedro y Pablo, no participan de una misma forma o esencia, sino que cada uno de ellos es numérica y esencialmente un individuo $(6,1,8)$. Obviamente, y de acuerdo con lo que ha dicho Suárez anteriormente, esta unidad formal ha de ser necesariamente incomunicable $(6,1,11)$. La comunidad, que recordemos necesita el universal, sólo puede proceder del entendimiento: "en las cosas singulares hay cierta semejanza en sus unidades formales, en la cual se funda la comunidad que el entendimiento puede atribuir a tal naturaleza en cuanto concebida por él" $(6,1,12)$.

Sólo desde estas premisas, y con lo dicho sobre las relaciones de razón y su importancia para el conocimiento científico, se alcanza una adecuada comprensión de lo que Suárez quiere decir al afirmar que "las naturalezas que llamamos universales y comunes son reales y existen verdaderamente en las cosas mismas" $(6,2,1)$. Pudiera parecer que tal afirmación está en las antípodas del pensar nominalista, pero el propio Suárez cree que en tal afirmación podrían coincidir los propios nominalistas. A estos sólo "puede censurárseles respecto de algunos modos de expresión, porque en realidad acaso no se apartan de la opinión verdadera [nam in re fortasse 
non dissident a vera sententia], ya que sus razones pretenden únicamente demostrar que la universalidad no se da en las cosas, sino que les conviene en cuanto están objetivamente en el entendimiento" $(6,2,1)$.

Las cosas que llamamos universales están en la realidad, pero la universalidad misma, como las relaciones de género y especie, sólo se halla en el entendimiento. Por eso los argumentos de los nominalistas "no son obstáculo alguno para aceptar como verdadero que las naturalezas denominadas universales existen en las cosas singulares y que estos singulares tienen algo en que convienen entre sí y se asemejan, y algo en que difieren o se distinguen" $(6,2,1)$.

Contra Courtine, no se trata aquí de que Suárez haya fraguado un nuevo concepto de realidad (recordemos al respecto que los entes de razón no son en sí mismos reales) ${ }^{21}$, sino de que está dotando de validez objetiva y científica a los entes de razón. Relaciones de razón con fundamento in re; ello es suficiente para asegurar la validez de la ciencia, contra los nominalistas más extremos que pretendían negarla. Respecto a ellos, escribe Suárez:

Por eso niegan falsamente que se hagan demostraciones o definiciones de las cosas, ya que las ciencias no tratan de los nombres y de nuestros conceptos formales, sino que tratan directamente de las cosas o de los conceptos objetivos. Por consiguiente, aunque la denominación de universalidad se aplique a las cosas en función de los conceptos, sin embargo, las cosas a las que denominamos así son reales y existen en el mundo real $(6,2,1)$.

A modo de conclusión, podemos mantener que el universal en Suárez se mueve totalmente en el ámbito de lo representable, de lo objetivo. En coherencia con la tendencia ontologista de la metafísica moderna, el orden causativo queda totalmente marginado de la cuestión: "porque no tratamos de lo que llaman universal 'causativo', o, a modo de eficiente o de ejemplar, sino del universal 'entitativo', o 'predicativo" $(6,2,3)$. Y tal universal no puede entenderse como "universal en acto", (como "naturaleza abstraída de toda individuación, concebida a modo de un ser indiviso y común a muchos y que posee aptitud inmediata para ser predicada de ellos" $(6,2,5)$ ), sino como "universal en potencia" (todo lo que "ofrece fundamento al entendimiento para que la pueda concebir o idear" $(6,2,5))$. Hay universal en potencia, porque puede darse en las cosas "algún fundamento de la abstracción o concepción universal realizada por el entendimiento" $(6,2,5)$. Y si a algo podemos llamar correctamente universal en acto, hay que referirlo siempre a la obra del entendimiento: "las naturalezas se convierten en universales en acto únicamente por

\footnotetext{
${ }^{21}$ Que Suárez no haya realizado aún este concepto de realidad no significa que no haya ya puesto sus bases conceptuales. Al respecto, comparto con Heidegger y Courtine la tesis de que Suárez ha dado pasos de gigante en la transformación de la metafísica de las causas en una ontología de las esencias conceptuales.
} 
obra del entendimiento, antecediéndole cierto fundamento por parte de las cosas mismas, razón por la que existen en la realidad universales en potencia" $(6,2,8)$.

Por todo ello, considero que la única manera de entender adecuadamente la relación de universalidad en Suárez es como un ente de razón, como una relación de razón con fundamento in re. Por si a alguien se le ocurre pensar que podría tratarse de una relación real de semejanza, Suárez considera tal relación como insuficiente para la razón de universal:

porque en virtud de esa semejanza, tomada precisivamente, bien en acto, bien en potencia, no se forma el concepto de algo como uno común a muchos, sino que se conciben muchas cosas como semejantes entre sí; y el universal en cuanto tal, debe ser concebido como algo uno. Además, porque el universal en cuanto universal es concebido como actualmente indiviso en cuanto tal y como aptitudinalmente divisible y comunicable; en cambio, las cosas, en cuanto semejantes por naturaleza en la realidad, mejor se diría que están actualmente divididas y que son aptitudinal o fundamentalmente unibles -por así decirlo- en una naturaleza concebida universalmente $(6,2,14)$.

Es decir, la relación real de semejanza fundaría, en todo caso, un conjunto de cosas similares, pero la unidad de concepto que requiere el universal no se contenta con esto, tiene que ir más allá y quiere un concepto uno pero aptitudinalmente divisible y comunicable, pero "la actitud para existir en muchos no conviene a la naturaleza común en sí con anterioridad al entendimiento" $(6,4,6)$.

Más adelante Suárez vuelve a remarcar que "la universalidad es obra del entendimiento con fundamento en la realidad [universalitas est per intellectum cum fundamento in re]" y que "de este modo se trata de cierta unidad de razón que conviene a las naturalezas tal como se ofrecen a nuestras mentes [atque ita esse quiamdam unitatem rationis, convenientem naturis prout obiiciuntur menti]" $(6,5,1)$.

Y a la pregunta de si los universales son entes, acabará respondiendo que la razón de universalidad en un ente de razón $(6,7,2)$. La manera de expresarse Suárez en estos pasajes es especialmente confusa porque se mueve en una línea peligrosa que puede llevarle a concebir la ciencia como algo inmanente, como mera representación. Suárez, en la cuerda floja, hace malabarismo para no traicionar el espíritu realista escolástico, al mismo tiempo que abre el espacio al ámbito de la objetividad creativa. La intención de universalidad, la universalidad misma, es un ente de razón, una relación de razón, pero apoyada en una naturaleza que existe en las cosas mismas, como vimos anteriormente.

\section{Suárez y las matemáticas}

Salta a la vista que cuando Suárez piensa en los entes de razón desde el punto de vista de su importancia para las ciencias, tiene en cuenta, fundamentalmente, las 
relaciones de razón que dan lugar a los conceptos universales. Se echa en falta, por lo tanto, un tipo de entes de razón que serán fundamentales para el desarrollo de la ciencia moderna: los matemáticos. De hecho, aunque escuetamente, la cuestión está planteada en las Disputationes. Así en la disputatio 54, concretamente en el parágrafo séptimo de la sección cuarta, Suárez afirmará que tanto el espacio, del que se ocupa la geometría, como la sucesión numérica, de la que se encarga la aritmética, son entes de razón. En concreto se trata de negaciones, ya que no hay un sujeto en acto para dichas carencias. En ningún lugar existe el espacio puro o el tiempo puro.

Y creo que en el hecho de que el trasfondo de lo matemático sea la negación encontramos la razón por la que Suárez aún no ha pensado nítidamente el sentido de una física matemática, el sentido de un mundo escrito en clave matemática. En el fondo, aun cuando tanto las negaciones como las relaciones son basales para el conocimiento científico, las relaciones de razón que se fundan directamente en lo real (con fundamento in re) contribuyen de una manera más directa al conocimiento que las negaciones que no se fundan directamente en lo real (sin fundamento in re); ocurriendo que las relaciones matemáticas son del segundo tipo (54,6,2-4). La ciencia sigue siendo pensada por Suárez aristotélicamente a partir de los grados de abstracción de la materia. El esquema sigue siendo antiplatónico y por lo tanto, en este aspecto, antimoderno. Finalmente, y en coherencia con lo dicho en el apartado anterior, el modelo de ciencia suareciano se mueve en un tipo muy concreto de relación con fundamento in re: el de la abstracción y su resultado, el universal.

En este punto consideramos interesante llevar a cabo una comparación entre la manera que tiene Suárez de tratar los entes de razón y la propuesta por Spinoza, claramente influenciada por la metafísica del primero ${ }^{22}$. En Spinoza los entes de razón fundamentales para las matemáticas no son negaciones, sino relaciones. Con ello dejan de estar en una situación de inferioridad desde el punto de vista de su utilidad para las ciencias.

Spinoza coincide con Suárez en la idea de que aunque el ente de razón no puede ser considerado en realidad un ser y por lo tanto no constituye el objeto propio de la metafísica, esta disciplina no puede dejar de hacerse cargo del importante lugar que ocupan en la fundamentación de las ciencias. Tan importante resulta el tema para Spinoza que con él abre precisamente sus Pensamientos metafísicos ${ }^{23}$, breve escrito donde analiza los tópicos fundamentales de su metafísica con clara influencia de Suárez. A este respecto, extrema la distinción entre la quimera y el ser ficti-

\footnotetext{
22 He tratado de forma más exhaustiva la influencia de Suárez en Spinoza en un trabajo presentado en el último congreso de la Sociedad Académica de Filosofía (celebrado en Tenerife entre los días 2 y 4 de febrero de 2011). El texto, pendiente de publicación, lleva por título "La presencia de Suárez en la metafísica de Spinoza".

23 B. Spinoza, Tratado de la reforma del entendimiento. Principios de la filosofía de Descartes. Pensamientos metafísicos, Madrid, Alianza, 2006 ( $1^{\mathrm{a}}$ ed. 1988; $1^{\mathrm{a}}$ ed. orig. 1663). Edición y traducción de Atilano Domínguez.
} 
cio, por un lado, y la negación y la relación, por el otro, al considerar que los primeros, de hecho, no constituyen entes de razón 24 . En realidad, lo que está haciendo Spinoza es mostrar la diferencia que hay entre los entes de razón importantes para el conocimiento (los entes de razón en sentido propio) y los que no lo son (quimeras y seres ficticios). Por otra parte, lo que llama "ente ficticio" coincide con la quimera de Suárez, mientras que la quimera para Spinoza es "aquello cuya naturaleza implica una abierta contradicción".

Respecto a los entes de razón, que por tanto engloban aquello que Suárez llama negaciones y relaciones de razón, Spinoza propone la siguiente definición: "el ente de razón no es más que un modo de pensar [una afección del entendimiento], que sirve para retener, explicar e imaginar más fácilmente las cosas entendidas" 25 .

Los entes de razón "no son ideas de cosas ni pueden ser considerados como tales, ni tienen tampoco ningún objeto que o exista necesariamente o pueda existir". Cuando investigamos las cosas reales, tenemos que tener esto muy presente, "pues una cosa es investigar la naturaleza de las cosas, y otra, los modos como nosotros las percibimos. Si confundimos una con otra, no podremos entender si los modos de percibir ni la naturaleza real" 26 . Con lo que caeremos en grandes errores. En esta distinción basaba, por ejemplo, su crítica al "prejuicio" de las causas finales en el apéndice al libro primero de la Ética.

Los entes de razón que sirven para retener las cosas entendidas coinciden con las relaciones universales de Suárez. Spinoza se refiere a los conceptos de género, especie, etc., y apunta a ellos como modos de pensar especialmente utilizados por los filósofos.

En cambio, desde el punto de vista de la importancia de los entes de razón para la ciencia, obviamente, Spinoza va a destacar los entes de razón matemáticos. Destaca el tiempo, el numero y la medida: "el tiempo sirve para explicar la duración, el número para la cantidad discreta y la medida para la cantidad continua". Estos son los entes de razón que sirven para explicar las cosas.

Además de estos dos tipos de entes de razón, que engloban aquello que Suárez llamaba relaciones de razón, Spinoza se refiere a las negaciones: "todos los modos de que se sirve el alma para negar, tales como la ceguera, la extremidad o fin, el término, las tinieblas, etc." 27 . Estos son, por último, los entes de razón o modos de pensar que sirven para que imaginemos las cosas.

\footnotetext{
24 Ibíd. pp. 244-245.

25 Ibíd. p. 242.

26 Ibíd. p. 245.

27 Ibíd. p. 243.
} 
Como conclusión podemos afirmar que en Suárez los entes de razón desempeñan un papel fundamental para el aseguramiento de la validez científica. Con ello el filósofo y teólogo granadino, y aunque aún no ha caído en la cuenta de la importancia de los entes de razón matemáticos para el desarrollo de la ciencia natural, ha asentado las bases de la forma barroca de entender el ámbito de la idealidad, aún no coincidente con el de la realidad, que sigue siendo pensada en términos fundamentalmente extramentales y, frente a los cuales, el ente de razón es sólo un artificio. Sólo cuando los entes ideales dejen de ser considerados como meros entes de razón, lo que hemos visto que ocurre ya claramente en Kant, se habrá consumado la equiparación de realidad e idealidad y, con ello, el giro ontologista y antropológico de la metafísica.

Óscar Barroso Fernández

obarroso@ugr.es

Universidad de Granada 